\title{
Value of a New QT Interval Correction Formula in Adjusting for Increases in Heart Rate
}

\author{
Simon W Rabkin* \\ Division of Cardiology, Department of Medicine, University of British Columbia, Canada
}

*Corresponding author: Dr. Simon W Rabkin, MD, FRCPC, FACC, FESC, Division of Cardiology, Department of Medicine, University of British Columbia, Level 92775 Laurel St, Vancouver, V5Z 1M9, BC, Canada, Tel: 604-875-5847, Fax: 604-8755849, E-mail: rabkin@mail.ubc.ca

\begin{abstract}
Background and objective: QT interval correction for heart rate $(\mathrm{QTC})$ is essential for clinical usage. A new $Q T c$ formula has been developed based on a data agnostic mathematical approach in a large population ECG data base. The formula requires testing on a within individual basis under conditions of intervention to increase heart rate. The objective of this study was to examine the stability and reliability the new QTc formulae within individuals when Heart Rate $(\mathrm{HR})$ is increased by dobutamine.
\end{abstract}

Methods: HR and QT interval were measured in fifteen individuals who received intravenous dobutamine in a sequential protocol at 5, 10, 20, 30 and 40 micrograms $/ \mathrm{kg} / \mathrm{min}$.

Results: Dobutamine produced a significant $(p<0.0001)$ increase in heart rate and a significant $(p<0.0001)$ reduction in QT. The new QTc formula showed relative stability with little change across the dobutamine dosages. Using a linear regression to evaluate the HR-QT interval, showed a significant $(p<0.0001)$ negative slope for the relationship. In contrast, the slope of the QTc-heart rate relationship for the new QTc formula did not differ from zero.

Conclusion: The new QT correction formula is relatively independent of the effect of increasing heart rate and should be useful QTc formula especially in conditions of increased heart rate.

\section{Keywords}

QT interval, QT heart rate correction formula, Dobutamine

\section{Introduction}

Assessment of the QT interval on the ECG has attracted considerable attention as a marker for potential drug-induced cardiac toxicity or drug-induced sudden cardiac death [1-3], electrolyte abnormalities [4], as well as the identification of individuals with genetic mutations that carry a high risk of sudden death [5]. A new QT-heart rate correction formula (QTc) has been developed based on ECGs from about 13,600 individuals in the NHANES US population study [6]. This QTc was shown to be was superior to other formulae $[6,7]$. The value of this approach, however, has not been tested in the same individual under different conditions of heart rate stress.

A critical aspect of the evaluation of the QT interval is its adjustment for heart rate because of the recognized inverse relationship between QT interval and heart rate. Studies that established QTc formulae including the most recent one, utilized data on different individuals who have different heart rates. While there is considerable power in the large numbers of individuals from population based studies, they do not address between individual variability. Specifically these studies are not able to take into consideration variables that produce different resting heart rates that might in turn influence the QT interval. The importance of the individual responses to test the robustness of the QT-heart rate correction formulae is well recognized [8]. The new QT-heart rate adjustment formula has not been subject to protocol driven changes in heart rate to determine whether QTc is stable when heart rate is increased. The objective of this study was to examine the new QT correction formula for its ability to remain stable in the same individual across a range of heart rates.

\section{Methods}

\section{Population}

The patient population were individuals who re-

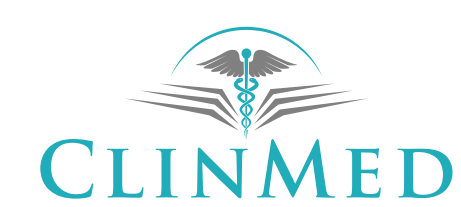

INTERNATIONAL LIBRARY
Citation: Rabkin SW (2017) Value of a New QT Interval Correction Formula in Adjusting for Increases in Heart Rate. Int J Clin Cardiol 4:101. doi.org/10.23937/2378-2951/1410101

Received: May 29, 2017; Accepted: August 01, 2017; Published: August 03, 2017

Copyright: () 2017 Rabkin SW. This is an open-access article distributed under the terms of the Creative Commons Attribution License, which permits unrestricted use, distribution, and reproduction in any medium, provided the original author and source are credited. 
ceived dobutamine for a MIBI study and whose baseline ECG showed sinus rhythm, no interventricular conduction delay (or bundle branch block) or significant ST-T changes. The study was approved by the Institution's Research Ethics Committee.

\section{Data collection}

Twelve-lead ECGs, recorded at a paper speed of 25 $\mathrm{mm} / \mathrm{s}$, were acquired in the supine position. Heart rate and QT measurements were made at specific time points during each stage of the dobutamine protocol. The first ECG was in the drug free, baseline condition, within $1 \mathrm{~min}$ before the beginning of dobutamine administration. The second ECG was at 2 min and 50 seconds after the first dose of dobutamine was started. Every subsequent ECG was taken $3 \mathrm{~min}$ after the increase in dobutamine concentration. The last ECG reported was 50 seconds after the fifth dose, or $15 \mathrm{~min}$ and $50 \mathrm{sec}$ after initial infusion. The dobutamine protocol consisted of intravenous infusion of dobutamine. Dobutamine was started at 5 micrograms/ $\mathrm{kg} / \mathrm{min}$ and increased at $3 \mathrm{~min}$ intervals to 10,20,30 and $40 \mathrm{micrograms} / \mathrm{kg} / \mathrm{min}$. In a few individual, the dose of dobutamine was stopped after 30 micrograms $/ \mathrm{kg} / \mathrm{min}$ when a marked tachycardia occurred.

The heart rate and QT interval were measured manually from the ECG. For each RR interval, a caliper was used to measure the distance from one peak of the QRS complex to the next peak. Each recorded RR value was the mean of two consecutive RR intervals. The distances measured were in $0.25 \mathrm{~mm}$ increments. The average of two measurements was used in all analysis. For each QT interval, a caliper was used to measure the distance from the start of the QRS complex to the end of the T wave. The end of the $T$ wave was defined as the intersection of a horizontal line representing the baseline of the ECG to the tangent of the downward curve of the $T$ wave at its steepest point. The ECG baseline is typically defined at the level of the PR interval. If the PR interval was very short and could not be easily used or absent, the beginning of the QRS complex was used. Each pair of corresponding RR and QT interval was taken from the same complex. The distances measured were in $0.25 \mathrm{~cm}$ increments, with an uncertainty of $\pm 0.25 \mathrm{~cm}$. As two readings were taken from each lead, the average of two was used in all analysis, The QT interval was almost always measured from lead II. Rarely, excessive baseline artifact (noise) in lead II, required QT measurement from leads I or III.

The basis for the formulation of the new QTc has been presented in detail [6]. Briefly, a spline correction function, modeled using a cubic regression spline with four knots and an adjustment for gender was fit to the QT and heart rate ECG data from 13,600 individuals in the US National Health and Nutrition Examination Survey (NHANES) II and III studies, conducted by the Center for Disease Control (CDC) of the US [6]. Considering the person's age, the spline QT correction, was developed. With each observation weighted by the respective
NHANES sampling weight with spline parameters selected as those that minimized the least squares estimate fit of the QT-heart rate relationship [6]. The ECG exclusion criteria were abnormalities that made calculation of the QT interval difficult such as right or left bundle branch block, myocardial infarction left ventricular hypertrophy or rhythm not being sinus [6].

\section{Data analysis}

Several approaches were used. In the first approach, all data from the ECG prior to and at the end of each dose of dobutamine were used. After completion of dobutamine infusion, QTC was compared. In the second approach, the slope of the relationship, between QTc and the different dobutamine dose was assessed. A linear least squares regression model was used to calculate the slope of QTc vs. heart rate relationship (GraphPad Prism). The data are presented as mean \pm 1SD. Statistical testing used repeated measures ANOVA to test variables across the different dobutamine doses. The relationship between QT and heart rate used regression analysis. Comparisons of the mean data for two different groups used the nonparametric Wilcoxon signed rank test.

\section{Results}

Dobutamine was associated with a significant $(p<$ 0.0001 ) increase in heart rate that increased from 74.3 $+11.6 \mathrm{bpm}$ (mean \pm SD) at baseline to $76.7+13.3,88.0$ $+16.3,104.0+20.7,113.2+21.0$ and $120.6+13.8 \mathrm{bpm}$ at respectively $5,10,20,30$ and 40 micrograms $/ \mathrm{kg} / \mathrm{min}$ of intravenous dobutamine (Figure 1). The magnitude of the change in heart rate was apparent (inset) and was significant ( $p<0.001$ ) as it increased by $48.6+12.0 \mathrm{bpm}$ from baseline to the highest dobutamine dose. There was a significant $(p<0.0001)$ reduction in QT interval

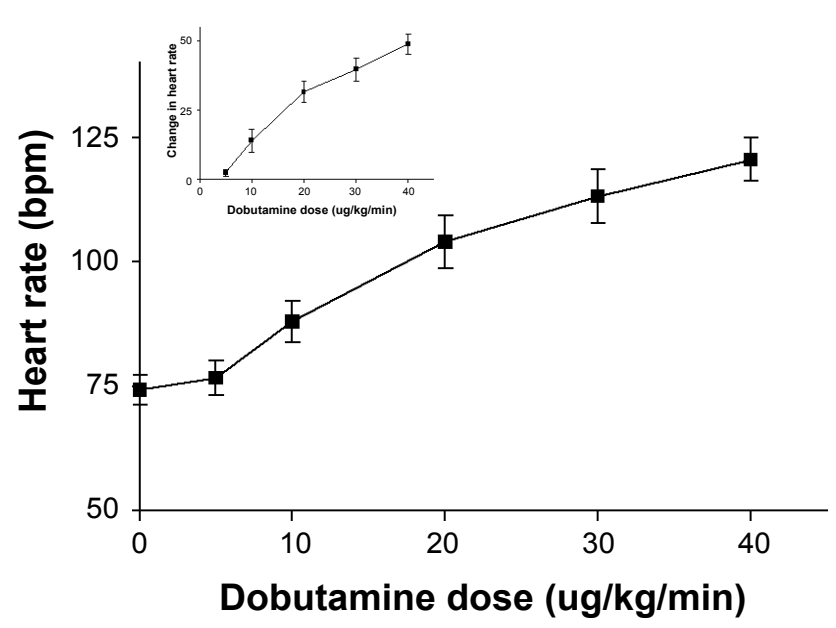

Figure 1: Shows the mean heart rate $( \pm S D)$ at the end of each dobutamine dose administration. The inset shows the change in heart rate from baseline to each of dobutamine doses. The sample size was the same at each dose $(n+15)$ except for the highest dose $(N=10)$. The same sample size was present in the following two figures. 


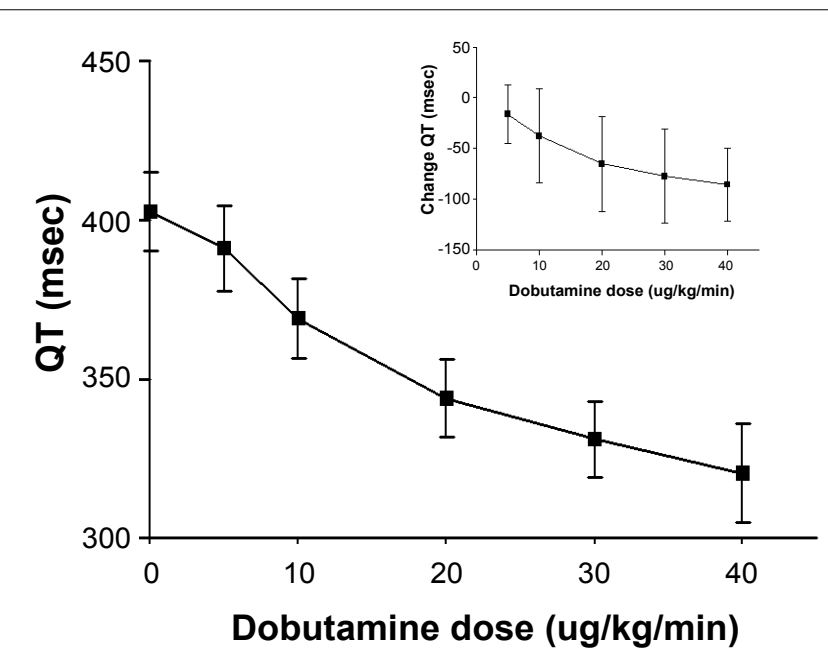

Figure 2: Shows the mean QT interval (+ SD) at the end of each dobutamine dose administration. The inset shows the change in QT interval from baseline to each of dobutamine doses.

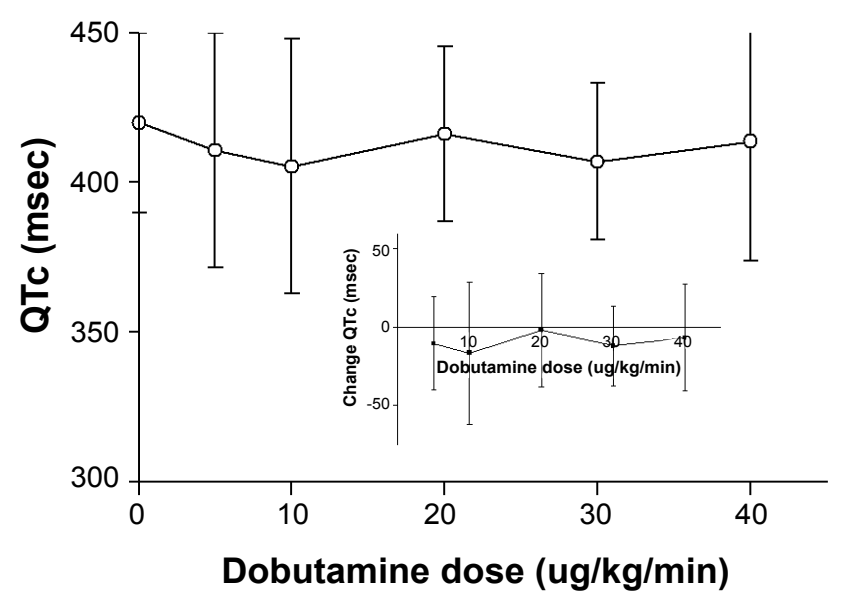

Figure 3: Shows the corrected QT interval (mean + SD) at the end of each dobutamine dose administration. The inset shows the change in corrected QT interval (mean + SD) at the end of each dobutamine dose administration.

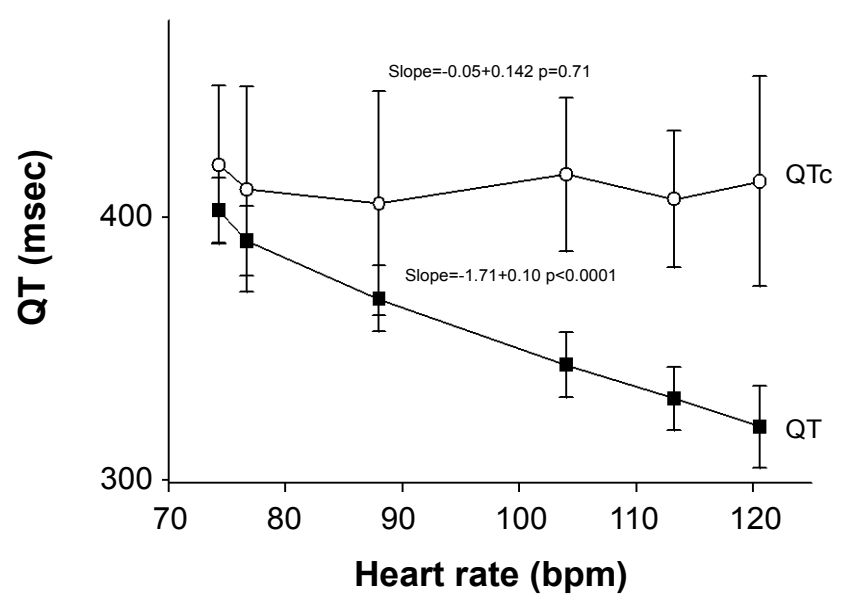

Figure 4: Shows the QT interval at the mean heart rate for each stage of the dobutamine dosages for uncorrected QT (solid squares) and QTc (open circles). with increasing dobutamine doses (Figure 2). The magnitude of the change in QT interval was apparent (inset) as the QT interval decreased significantly $(p=0.008)$ by $85.3+36.2 \mathrm{msec}$ from baseline to the highest dobutamine dose.

The new QTc was not significantly different at the different dobutamine dosages and there was no significant change in QTC across the different dobutamine doses (Figure 3).

The heart rate QT relationship was explored in two ways. First the QT interval at the mean heart rate for each stage of the dobutamine dosages was examined (Figure 4). There was a significant negative correlation between the two factors as the QT interval reduction was associated with increasing heart rates. In contrast, there was no such relationship for the new QTc formula.

Because the relationship of $Q T$ to heart rate can vary between individuals [8], this relationship was examined for each individual. This is exemplified in one case in which the QT interval at each heart rate is displayed. The significant negative correlation between QT and heart rate was eliminated by the new correction formula (Figure 5). QT interval at each heart rate for each individual was examined (Figure 6). Because the goal of an adjustment formula is to produce QTc values that are not correlated with heart rate i.e. the slope of QTc/RR regression being zero, the linear slope for each individual was calculated. The mean value for the QTc was close to zero i.e. $-0.20+0.58 \mathrm{msec} / \mathrm{bpm}$ and was significantly $(p<0.001)$ less that the uncorrected QT which had a slope of $-1.89+0.56 \mathrm{msec} / \mathrm{bpm}$.

\section{Discussion}

This is the first study to test the newer QT correction formula in the setting of intra-individual changes in heart rate. The increase in heart rate was induced by catecholamines. The new formula was found to be relatively independent of heart rate. These data suggest that this QT correction formula should of considerable value in identifying drug toxicity and electrolyte imbalance in patients with increases in heart rate $[1,2,4,5]$. QT heart rate adjustment formulae, including the one used herein, were been developed after examination of the QT intervals in different persons with different heart rates. This kind of analysis includes between person variability of QT assessment in addition to considerations of QTheart rate relationship. Thus there is concern about the extent to which between individual variability influences the validity of a QTc formula i.e. persons with faster heart rates may have different myocardial characteristics that are determinants of QT duration, compared to persons with slower heart rates [8]. Instead the present study evaluated the change in heart rate in the same individual and examined its impact on the QT interval.

Two kinds of data analysis were used-an assessment of group data at each dobutamine dose and an assess- 

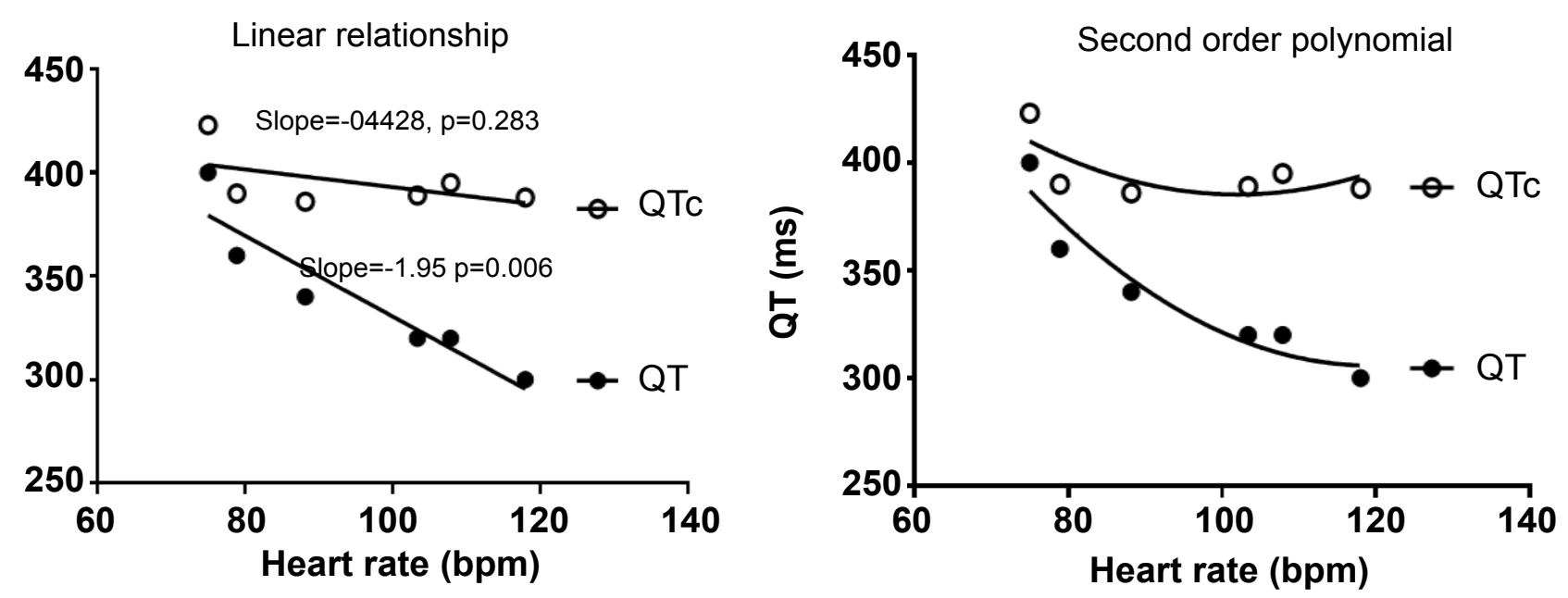

Figure 5: Shows a case with the QT interval for heat heart rate for uncorrected QT (solid squares) and QTc (open circle). The linear slope for each relationship (QT-Heart rate) is shown along with the significance level indicating whether the slope of the relationship is different from zero i.e. no relationship between QT interval and heart rate.
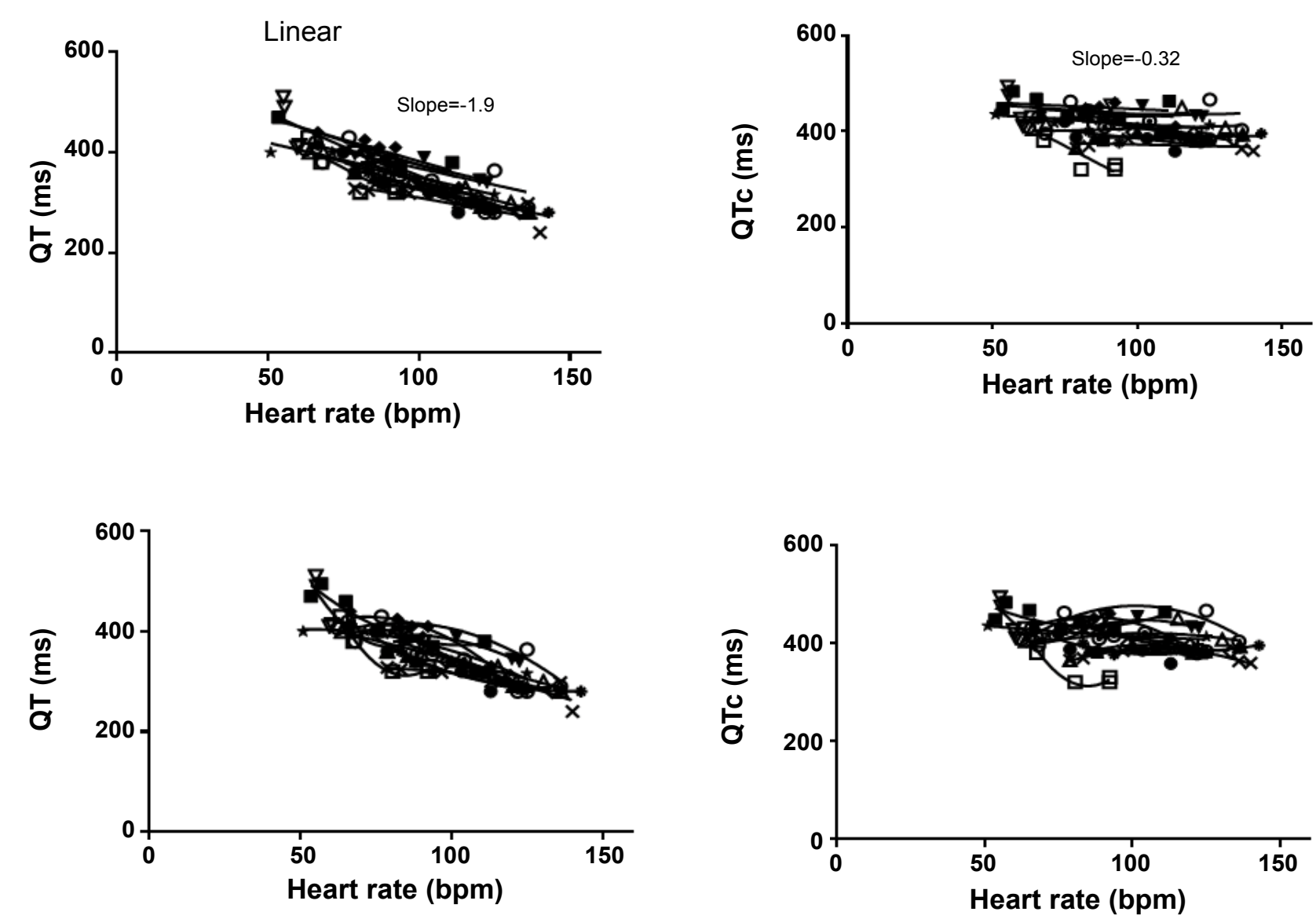

Figure 6: Shows the QT interval at each heart rate for each individual and each line represents the relationship for each individual with the QT interval (left panel) and corrected QT interval (right panel). The linear slope QT-Heart rate is shown for all the individuals and represents the mean + SD from each individual.

ment of the change in QT with change in heart rate for each individual. Using the first approach there were little differences in the QTc with increases in heart rate. Because an individual data approach has been proposed as the optimal one to adjust for changes in QTc [8], the slope of the QTc-heart rate relationship was evaluated in each individual as heart rate was increased. The in- verse correlation between QT interval and heart rate was obliterated by the new QTc formula.

Heart rate was increased by administration of dobutamine. This study elucidates the differences of opinion on the effects of dobutamine on QTc in the very few studies that have previously commented on this question [9-11]. The present study showed no significant effect of dobu- 
tamine on QT interval once the QT interval has been corrected for the effect of heart rate. Dobutamine-induced stress cardiomyopathy was reported to be associated with prolonged QTc but the kind of correction formula was not specified [12]. This data also has specific relevance to the assessment of the QT interval in situations of catecholamine administration or excess. Catecholamine administration has been proposed as a diagnostic tool to unmask concealed long QT Syndrome [13]. Hyper adrenergic states are present in a number of clinical conditions and those associated with psychiatric conditions or in critically ill patients where the measurement of QT interval is an important consideration for assessment of drug toxicity or electrolyte abnormalities $[2,4,14]$.

\section{Limitations}

There are several limitations of this study that warrant further discussion. First, the sample size was relatively small. Dobutamine is a relatively infrequently used method to induce myocardial stress for imaging so that such cases are infrequent. Second, the effects of dobutamine entails considerations of its kinetics in the circulation (pharmacokinetics) and binding to relevant cardiac receptors which are manifested as immediate responses as well as a mono-exponential response to a new steadystate value $[11,15]$. Third, evaluation of a new QT correction formulae confront the challenge of the absence of a true (gold) standard against which a new formula can be judged. Fourth, there was no correction for QT/RR hysteresis i.e. the QT interval duration is not only dependent on the simultaneously measured RR intervals but also on the prior RR intervals. How quickly the QT interval adapts to heart rate changes and how long is the 'memory' of previous $R R$ intervals stored in the myocardium is difficult to ascertain with the dobutamine infusion protocol which involves a stepwise increase in dobutamine concentration in order to ensure significant increases in heart rate. Auto-regression equations have been developed to minimize hysteresis but they require prolonged recordings at steady state [16]. In the absence of such prolonged observation periods, investigators have suggested using the heart rate correction formula at a fixed time interval [16] such was used in the protocol in this study. The QT interval in the present study was measured after 3 min of dobutamine infusion at each dosage.

\section{Conclusions}

The newer QT-heart rate correction formula provides a very good adjustment for increases in heart rate. Clinical practice should consider switching to the newer formula especially in conditions of faster heart rates.

\section{Conflicts of Interest}

None.

\section{References}

1. Kannankeril P, Roden DM, Darbar D (2010) Drug-induced long QT syndrome. Pharmacol Rev 62: 760-781.
2. Rabkin SW (2014) Aging effects on QT interval: Implications for cardiac safety of antipsychotic drugs. J Geriatr Cardiol 11: 20-25.

3. U.S. Department of Health and Human Services Food and Drug Administration (2005) Clinical Evaluation of QT/ QTc Interval Prolongation and Proarrhythmic Potential for Non-Antiarrhythmic Drugs.

4. Whitted AD, Stanifer JW, Dube P, Borkowski BJ, Yusuf J, et al. (2010) A dyshomeostasis of electrolytes and trace elements in acute stressor states: impact on the heart. Am J Med Sci 340: 48-53.

5. Bokil NJ, Baisden JM, Radford DJ, Summers KM (2010) Molecular genetics of long QT syndrome. Mol Genet Metab 101: 1-8.

6. Rabkin SW, Szefer E, Thompson DJS (2017) A new QT interval correction formulae to adjust for increases in heart rate. JACC: Clinical Electrophysiology 3: 756-766.

7. Rabkin SW (2017) Criteria for short QT interval based on a new QT heart rate adjustment formula. J of Arrhythmia.

8. Manion CV, Whitsett TL, Wilson MF (1980) Applicability of correcting the QT interval for heart rate. AHJ American Heart Journal 99: 678.

9. Dahl EK, Moller S, Kjaer A, Petersen CL, Bendtsen F, et al. (2014) Diastolic and autonomic dysfunction in early cirrhosis: a dobutamine stress study. Scand J Gastroenterol 49: 362-372.

10. Papadopoulos C, Zaglavara T, Karvounis HI, Haaverstad R, Parharidis GE, et al. (2006) QT dispersion is determined by the relative extent of normal, hibernating, and scarred myocardium in patients with chronic ischemic cardiomyopathy. A dobutamine stress echocardiography study before and after surgical revascularization. Journal of Electrocardiology 39: 103-109.

11. Seethala S, Shusterman V, Saba S (2011) Effect of beta-adrenergic stimulation on QT interval accommodation. Heart Rhythm 8: 263-270.

12. Abraham J, Mudd J, Kapur N, Klein K, Champion HC, et al. (2009) Stress cardiomyopathy after intravenous administration of catecholamines and beta-receptor agonists. J Am Coll Cardiol 53: 1320-1325.

13. Vyas H, Ackerman MJ (2006) Epinephrine QT stress testing in congenital long QT syndrome. J Electrocardiol 39: S107-S113.

14. Kalk N, Nutt D, Lingford-Hughes AR (2011) The role of central noradrenergic dysregulation in anxiety disorderss: evidence from clinical studies. J Psychopharmacol 25: 3-16.

15. Ruffolo RR Jr (1987) The pharmacology of dobutamine. Am J Med Sci 294: 244-248.

16. Jacquemet $V$, Dube $B$, Knight $R$, Nadeau $R$, LeBlanc $A R$, et al. (2011) Evaluation of a subject-specific transfer- function-based nonlinear QT interval rate-correction method. Physiol Meas 32: 619-635. 